\title{
Comprehensive Analysis of Genomic Safe Harbors as Target Sites for Stable Expression of the Heterologous Gene in HEK293 Cells
}

Seunghyeon Shin ${ }^{1}$, Su Hyun Kim ${ }^{1}$, Sung Wook Shin², Lise Marie Grav ${ }^{3}$, Lasse Ebdrup Pedersen ${ }^{3}$, Jae Seong Lee ${ }^{2}$, Gyun Min Lee ${ }^{1,3}$

${ }^{1}$ Department of Biological Sciences, KAIST, Daejeon, Republic of Korea

${ }^{2}$ Department of Molecular Science and Technology, Ajou University, Suwon, Republic of Korea

${ }^{3}$ The Novo Nordisk Foundation Center for Biosustainability, Technical University of Denmark, Kongens Lyngby, Denmark

\section{Correspondence}

Jae Seong Lee, Department of Molecular Science and Technology, Ajou University, Suwon 16499, Republic of Korea.

E-mail: jaeseonglee@ajou.ac.kr

Gyun Min Lee, Department of Biological Sciences, KAIST, 291 Daehak-ro, Yuseong-gu, Daejeon 305-701, Republic of Korea.

E-mail: gyunminlee@kaist.ac.kr 


\begin{tabular}{|c|c|}
\hline $\begin{array}{c}\text { Methods, Figures and } \\
\text { Tables }\end{array}$ & Page number \\
\hline Methods & 3,4 \\
\hline Supporting Figure S1 & 5,6 \\
\hline Supporting Figure S2 & 7 \\
\hline Supporting Figure S3 & 8 \\
\hline Supporting Figure S4 & 9,10 \\
\hline Supporting Figure S5 & 11 \\
\hline Supporting Table S1 & $12,13,14$ \\
\hline Supporting Table S2 & 15,16 \\
\hline Supporting Table S3 & $17,18,19,20$ \\
\hline Supporting Table S4 & $21,22,23$ \\
\hline
\end{tabular}




\section{Methods}

\section{5'/3' junction PCR and qRT-PCR for stable clones harboring eGFP transgene}

Genomic DNA had been extracted from cell pools and stable clones in 96-wells using a QuickExtract DNA extraction solution (Epicentre, Illumina, Madison, WI), as previously described..$^{1}$ The 5'/3' junction PCR was performed using PrimeSTAR HS Premix (Takara Bio, Shiga, Japan) by touchdown PCR $\left(98^{\circ} \mathrm{C}\right.$ for $3 \mathrm{~min}$; $10 \times$ : $98^{\circ} \mathrm{C}$ for $10 \mathrm{sec}, 68^{\circ} \mathrm{C}-58^{\circ} \mathrm{C}\left(-1^{\circ} \mathrm{C} /\right.$ cycle $)$ for $30 \mathrm{sec}, 72^{\circ} \mathrm{C}$ for $2 \mathrm{~min}$; $30 \times$ : $98^{\circ} \mathrm{C}$ for $10 \mathrm{sec}, 58^{\circ} \mathrm{C}$ for $30 \mathrm{sec}, 72^{\circ} \mathrm{C}$ for $2 \mathrm{~min} ; 72^{\circ} \mathrm{C}$ for $10 \mathrm{~min}$ ). The genomic DNA preparation process and copy number analysis were conducted, as previously described. ${ }^{2}$ The calculation of the relative eGFP gene copy number was based on the $\Delta \Delta \mathrm{CT}$ method, normalized to the human ACTB gene. ${ }^{3,4}$ The primer sequences for qRT-PCR are shown in Supporting Information Table S3.

\section{TLA sequencing of the A18 clone}

The A18 clone, which harbors the transgene at AAVS1 locus, was analyzed by TLA sequencing. Using two primer sets, the CMV promoter or eGFP sequence and/or proximal genomic DNA was amplified. By sequencing amplified library, TLA sequence coverage across the transgene integration locus was obtained, and remapping of TLA data identified the breakpoint sequences between transgene and genomic DNA. The primer sequences for TLA sequencing are shown in Supporting Information Table S3.

\section{Generation of CMV D.C. clones}

To generate the promoter-deleted clones, sgRNA-Cas9 vectors that target the 5' and 3' ends of the CMV promoter were constructed. Information on the sgRNA target site and plasmids are summarized in Supporting Information Tables S1,2. The primers for PCR and amplicon sequencing are listed in Supporting Information Table S3. 


\section{Plasmid construction for landing pad and generation of MCL}

The donor plasmid with LP was constructed using the USER cloning method. ${ }^{1}$ Information on plasmids and primers used in the USER cloning are listed in Supporting Information Tables S2,3. The 5' and 3' homology arm, puromycin resistance gene, and backbone sequences were obtained from the AAVS1-eGFP donor plasmid. The LP sequence was amplified from the LP donor plasmid comprising the EF1 $\alpha$ promoter and mCherry-coding sequences, which are flanked by loxP at the 5' end and lox2272 at the $3^{\prime}$ end. $^{5}$ The HEK293E host cells at $1.0 \times 10^{6}$ cells $/ \mathrm{mL}$ were transfected using Lipofectamine 2000 (Invitrogen, Carlsbad, CA), with the sgRNA-Cas9 vector which targets the AAVS1 locus, and the LP donor plasmid, which contains the EF1 $\alpha$ promoter and mCherry-coding sequence at a ratio of 1:1 (w:w). The stable cell pools were generated with $3 \mu \mathrm{g} / \mathrm{mL}$ of puromycin (Sigma-Aldrich, St. Louis, MO) selection for 2 weeks and stable clones were established by limiting dilution.

\section{Plasmid construction for RMCE donor and generation of recombinant cell pool}

Four RMCE donor plasmids (CMV-ETN, EF1 $\alpha$-ETN, CMV-EPO, and EF1 $\alpha$-EPO) were constructed. The backbone sequence for the RMCE donor plasmid was amplified from the RMCE EPO donor plasmid. ${ }^{5}$ The primer sequences, and information on the promoter and GOI are shown in Supporting Information Tables S3,4, respectively. The MCLs at $1.0 \times 10^{6}$ cells $/ \mathrm{mL}$ were transfected with a donor plasmid and a Cre recombinase vector (Sigma-Aldrich; OGS591) at a ratio of 5:1 (w:w) using Lipofectamine 2000 (Invitrogen). After 14 days, cell pools were sorted by FACS Aria II (BD Biosciences) to separate the mCherry-negative population. 
(a)

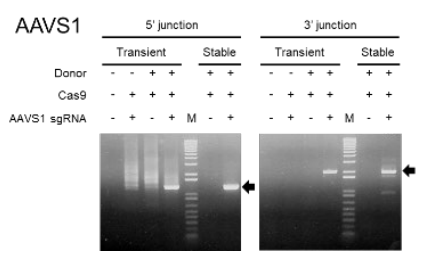

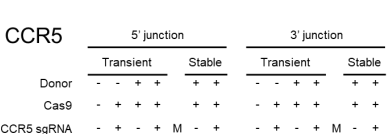
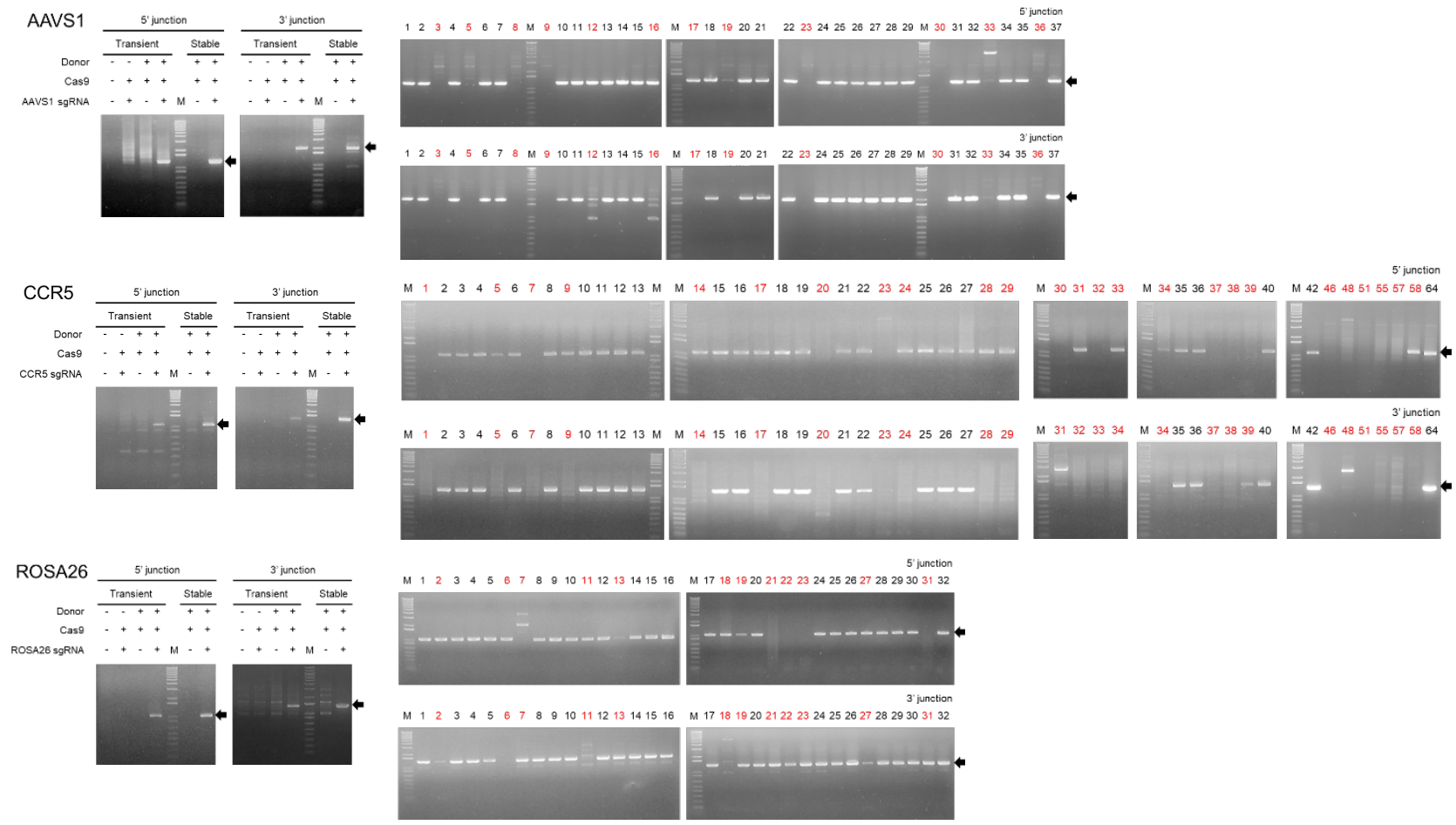

(b)

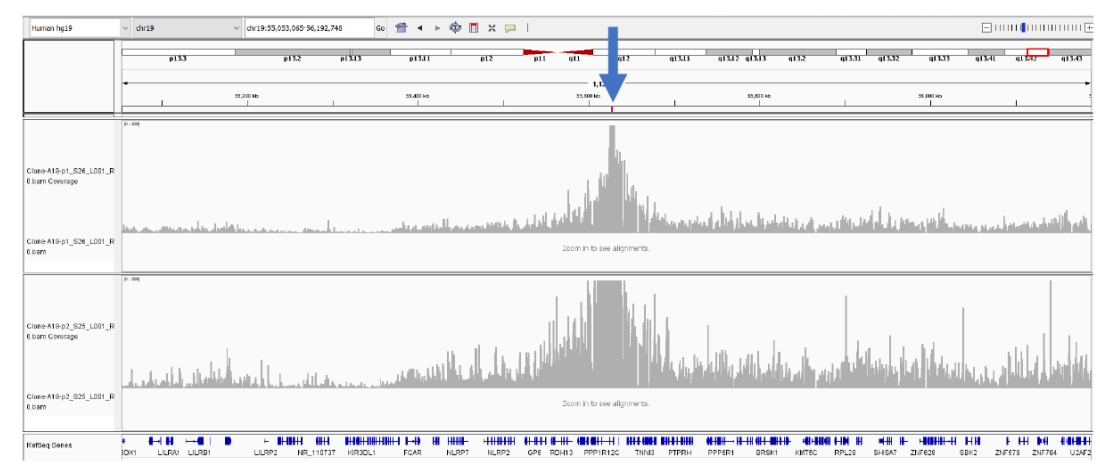

(c)
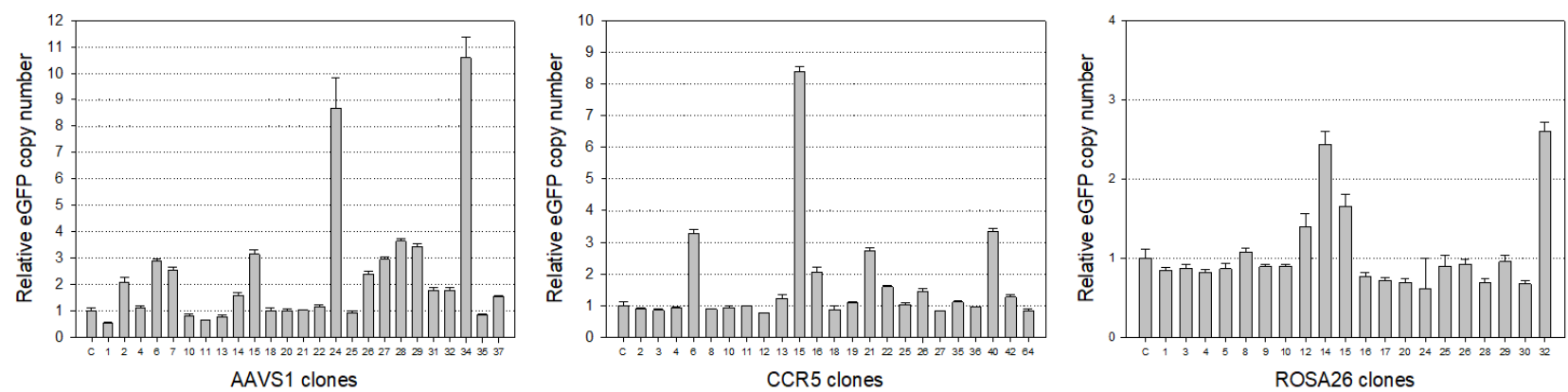
Supporting Figure S1. Generation of stable clones that express eGFP at GSHs by site-specific integration. (a) Verification of targeted integration at a designated locus by 5 '/3' junction PCR. Numerals represent the number of stable clones, and the junction PCR-negative clones are shown in red. M, 1-kb DNA ladder. The black arrow indicates the expected size of the amplicons. The targeting efficiencies for precise integration in stable clones were calculated by dividing the number of bothjunction positive stable clones by the number of total stable clones, which were $67.6 \%$ (25 out of 37 ) for AAVS1, $47.9 \%$ (23 out of 48) for CCR5, and 62.5\% (20 out of 32) for ROSA26 locus. (b) TLA sequencing of the A18 clone. Sequence coverage (in gray) was generated with primer set 1 (CMV, top) and primer set 2 (eGFP, bottom). The blue arrow indicates the breakpoint sequences identified with both primer sets. The red bar indicates the homology arms. The Y-axis is limited to 200x. (c) Measurement of the relative eGFP copy number in stable clones. The stable clones, which harbor one copy of the transgene at a designated locus, had values that range from 0.5 to 1.5 based on the qRTPCR results, which were $44.0 \%$ (11 out of 25 ) for AAVS1, $73.9 \%$ (17 out of 23 ) for CCR5, and $85.0 \%$ (17 out of 20) for ROSA26 locus. The error bars represent the standard deviations of three independent experiments. 
(a)

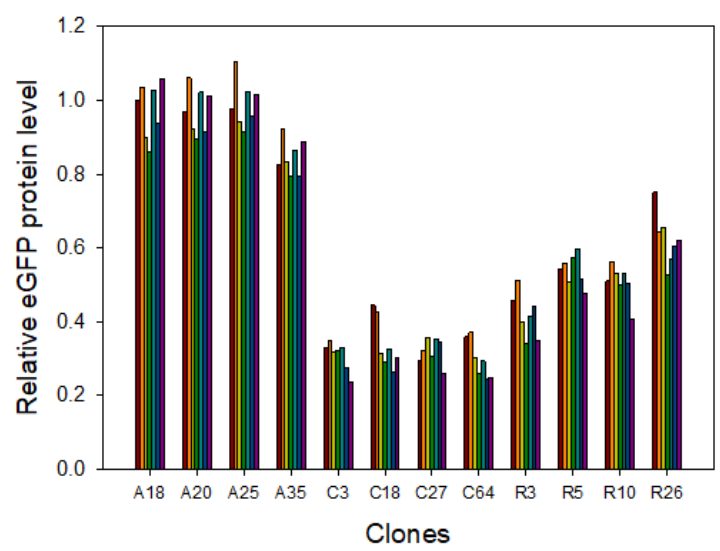

(c)

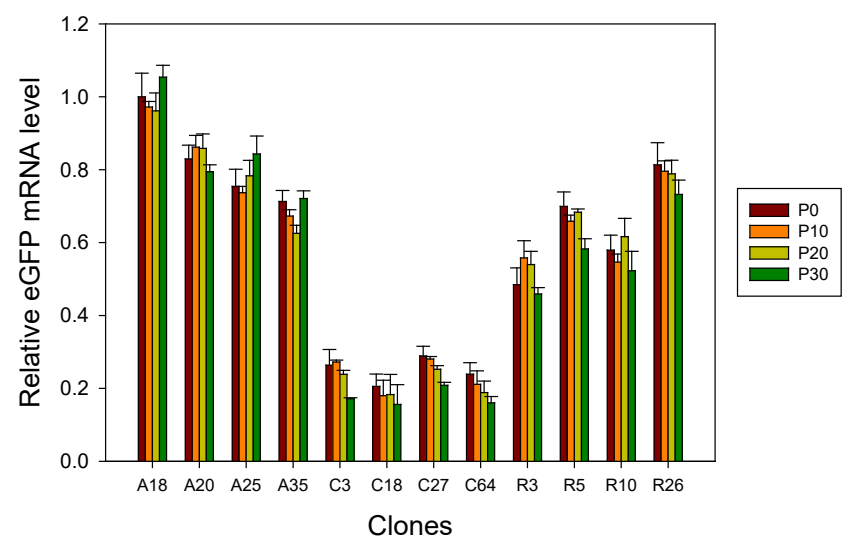

(b)

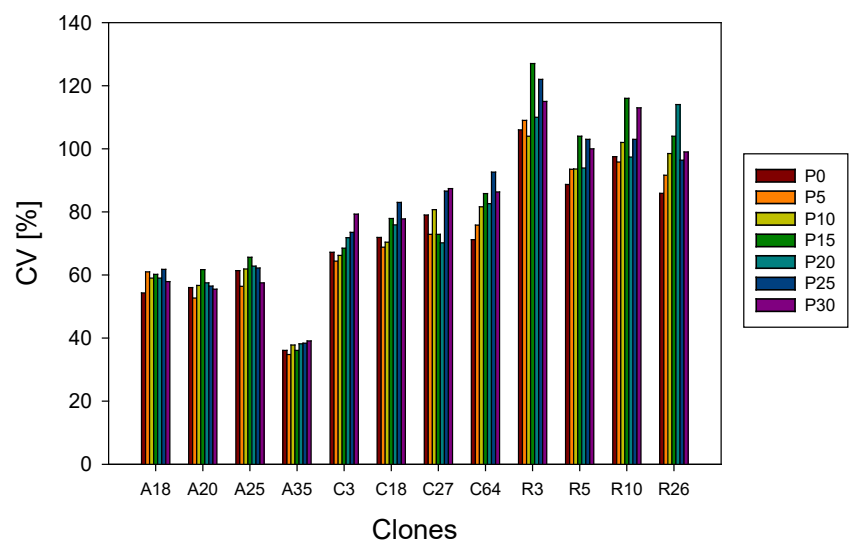

Supporting Figure S2. Stability of the eGFP transgene expression at GSHs during the long-term culture. (a) Change in the relative eGFP protein levels of the stable clones. The eGFP protein levels were measured by flow cytometry at passage 0 (P0), 5 (P5), 10 (P10), 15 (P15), 20 (P20), 25 (P25), and 30 (P30) and was normalized to A18 at P0. (b) Change in the CV values of the stable clones. The CV value was measured by flow cytometry at P0, P5, P10, P15, P20, P25, and P30. (c) Change in the relative eGFP mRNA levels of the stable clones. The mRNA levels were measured by qRT-PCR at P0, P10, P20, and P30, and was normalized to A18 at P0. The error bars represent the standard deviations of the three independent experiments. 
(a)

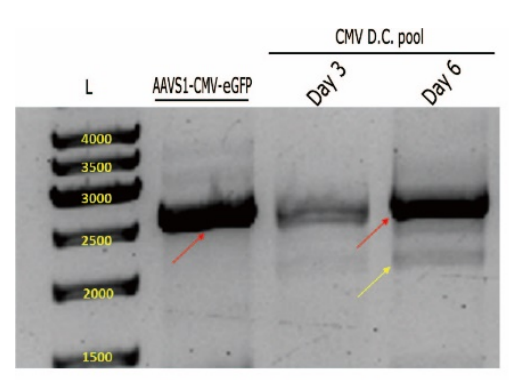

Expected size $=2,600 \mathrm{bp}(\mathrm{WT}), 2,069 \mathrm{bp}(D . C$.

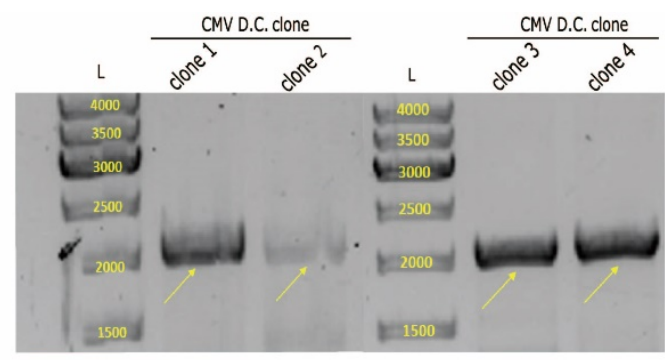

Expected size $=2,069 \mathrm{bp}(D . C$.)

(b)

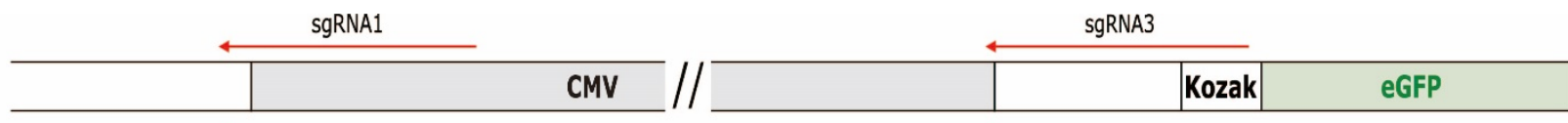

\begin{tabular}{|c|c|}
\hline AAVS1-CMV-eGFP & ........CCGCGTTACATAACTTACGGTAAATGGCCCGCCTGG........//........TGGTTAGTGAACCGTCAGATCCGCTAGCGCTACCGGTCGCCACCATGGTGAGCAAGGGCGAGGAGCTG.. \\
\hline CMV D.C. clone 1 & GCGCTACCGGTCGCCACCATGGTGAGCAAGGGCGAGGAGCTG. \\
\hline CMV D.C. clone 3 & GCGCTACCGGTCGCCACCATGGTGAGCAAGGGCGAGGAGCTG.. \\
\hline CMV D.C. clone 4 & ........CCGCGT \\
\hline
\end{tabular}

-...-Deletion

Supporting Figure S3. Generation of CMV D.C. stable clones from the A18 clone, using CRISPR/Cas9-mediated double-strand breaks. (a) Verification of the CMV promoter deletion by PCR in the cell pool and stable clones. Red arrows indicate wild type band, without CMV promoter deletion, while yellow arrows indicate the D.C. band. (b) Validation of CMV D.C. clone 1, 3, and 4 by amplicon sequencing. PCR amplicons comprising the deletion sequence were analyzed by Sanger sequencing. The PAM sequences of target site are shown in red, while sgRNA-matching sequences are shown in blue. 
(a)

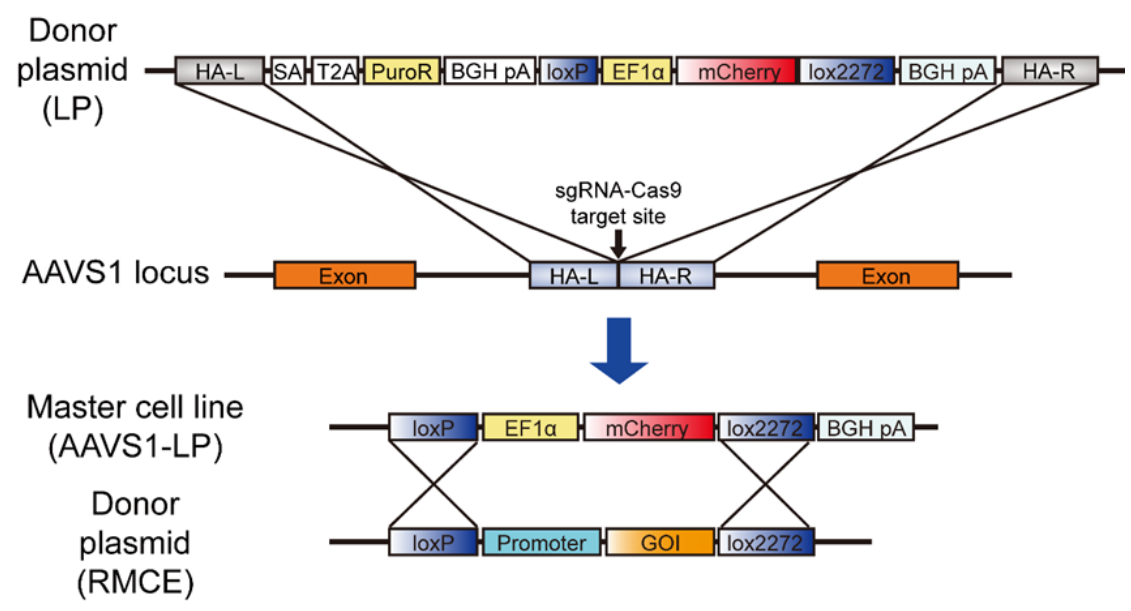

(b)

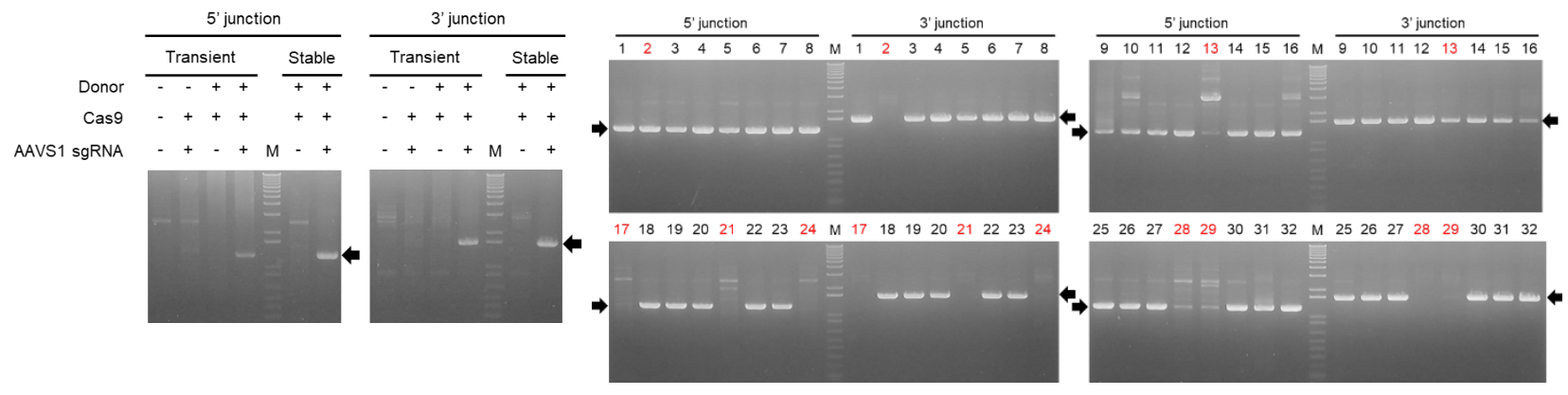

(c)

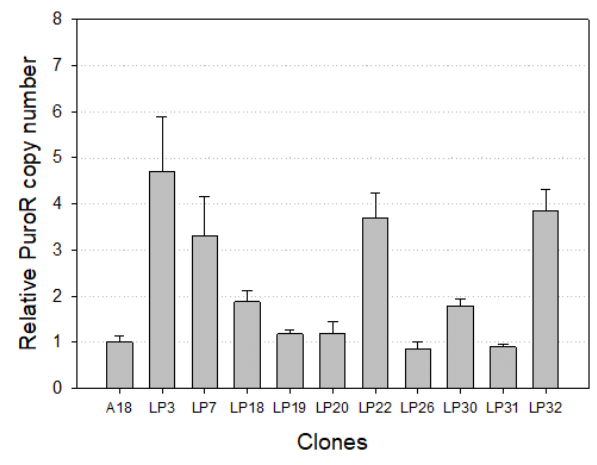

Supporting Figure S4. Generation of MCL with a landing pad (LP) at the AAVS1 locus by CRISPR/Cas9-mediated targeted integration. (a) A schematic diagram of the MCL generation that harbors LP at the AAVS1 locus, and the recombination process with the RMCE donor plasmid. (b) Verification of targeted integration of the LP sequence at the AAVS1 locus by $5^{\prime} / 3^{\prime}$ junction PCR. 
Numerals represent the number of stable clones, while the junction PCR-negative clones are shown in red. M, 1-kb DNA ladder. The black arrow indicates the expected size of the amplicons. (c) The copy number of the puromycin resistance gene was compared to the A18 clone, which was confirmed by targeted locus amplification and sequencing. The error bars represent the standard deviations of three independent experiments. 

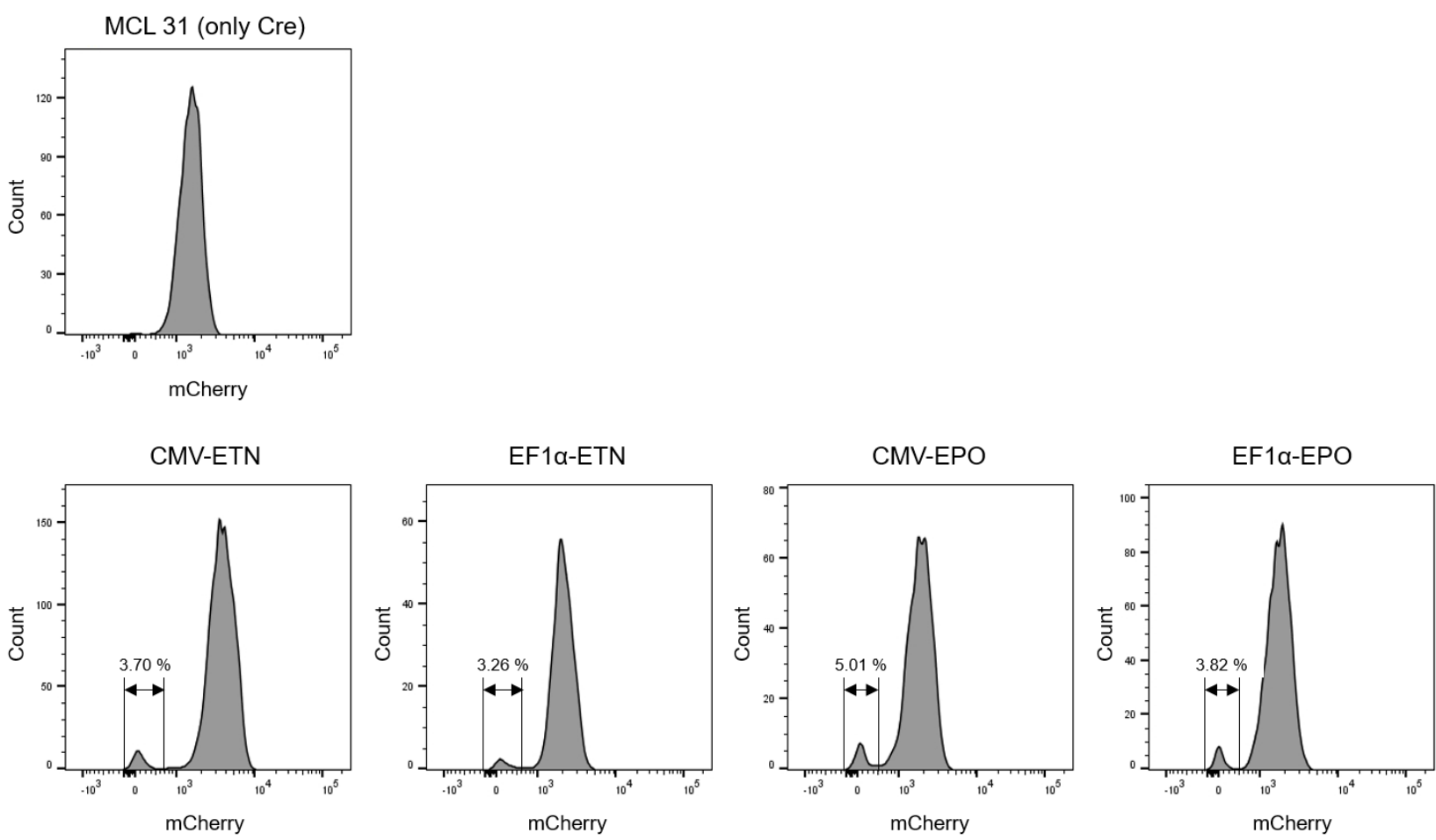

Supporting Figure S5. Generation of a subpopulation that produces ETN or EPO by RMCE and bulk sorting. The mCherry-negative cells were gated with MCLs that were transfected with only Cre recombinase. Numbers represent the percentage of populations of which LP was replaced by GOI. 
Supporting Table S1. sgRNA target sites and 5'/3' homology arm sequences.

\begin{tabular}{|c|c|c|c|}
\hline $\begin{array}{l}\text { Target } \\
\text { site }\end{array}$ & $\begin{array}{c}\text { sgRNA } \\
\text { target } \\
\text { sequence } \\
\text { (N20NGG) } \\
\end{array}$ & 5' homology arm sequence & 3' homology arm sequence \\
\hline $\mathrm{AAVS}^{6}$ & $\begin{array}{c}\text { ACCCCACA } \\
\text { GTGGGGCC } \\
\text { ACTAGGG }\end{array}$ & $\begin{array}{l}\text { CCGTCTCTCTCCTGAGTCCGGACCA } \\
\text { CTTTGAGCTCTACTGGCTTCTGCGC } \\
\text { CGCCTCTGGCCCACTGTTTCCCCTTC } \\
\text { CCAGGCAGGTCCTGCTTTCTCTGAC } \\
\text { CTGCATTCTCTCCCCTGGCTGTG } \\
\text { CCGCTTTCTGTCTGCAGCTTGTGGC } \\
\text { CTGGGTCACCTCTACGGCTGGCCCA } \\
\text { GATCCTTCCCTGCCGCCTCCTTCAG } \\
\text { GTTCCGTCTTCCTCCACTCCCTCTTC } \\
\text { CCCTTGCTCTCTGCTGTGTTGCTGCC } \\
\text { CAAGGATGCTCTTTCCGGAGCACTT } \\
\text { CCTTCTCGGCGCTGCACCACGTGAT } \\
\text { GTCCTCTGAGCGGATCCTCCCCGTG } \\
\text { TCTGGGTCCTCTCCGGGCATCTCTC } \\
\text { CTCCCTCACCCAACCCATGCCGTC } \\
\text { TTCACTCGCTGGGTTCCCTTTTCCTT } \\
\text { CTCCTTCTGGGGCCTGTGCCATCTCT } \\
\text { CGTTTCTTAGGATGGCCTTCTCCGA } \\
\text { CGGATGTCTCCCTTGCGTCCCGCCT } \\
\text { CCCCTTCTTGTAGGCCTGCATCATC } \\
\text { ACCGTTTTTCTGGACAACCCCAAAG } \\
\text { TACCCCGTCTCCCTGGCTTTAGCCA } \\
\text { CCTCTCCATCCTCTTGCTTTCTTGC } \\
\text { CTGGACACCCCGTTCTCCTGTGGAT } \\
\text { TCGGGTCACCTCTCACTCCTTTCATT } \\
\text { TGGGCAGCTCCCCTACCCCCCTTAC } \\
\text { CTCTCTAGTCTGTGCTAGCTCTTCCA } \\
\text { GCCCCCTGTCATGGCATCTTCCAGG } \\
\text { GGTCCGAGAGCTCAGCTAGTCTTCT } \\
\text { TCCTCCAACCCGGGCCCCTATGTCC } \\
\text { ACTTCAGGACAGCATGTTTGCTGCC } \\
\text { TCCAGGGATCCTGTGTCCCCGAGCT } \\
\text { GGGACCACCTTATATTCCCAGGGCC } \\
\text { GGTTAATGTGGCTCTGGTTCTGGGT } \\
\text { ACTTTTATCTGTCCCCTCCACCCCAC } \\
\text { AGT }\end{array}$ & $\begin{array}{l}\text { GGGGCCACTAGGGACAGGATTGGT } \\
\text { GACAGAAAAGCCCATCCTTAGGCC } \\
\text { TCCTCCTTCCTAGTCTCCTGATATTG } \\
\text { GGTCTAACCCCCACCTCCTGTAGG } \\
\text { CAGATTCCTATCTGGTGACACACC } \\
\text { CCCATTTCCTGGAGCCATCTCTCTCC } \\
\text { TTGCCAGAACCTCTAAGGTTTGCTT } \\
\text { ACGATGGAGCCAGAGAGGATCCTG } \\
\text { GGAGGGAGAGCTTGGCAGGGGGT } \\
\text { GGAGGGAAGGGGGGGATGCGTGAC } \\
\text { CTGCCCGGTTCTCAGTGGCCACCCT } \\
\text { GCGCTACCCTCTCCCAGAACCTGAG } \\
\text { CTGCTCTGACGCGGCCGTCTGGTGC } \\
\text { GTTTCACTGATCCTGGTGCTGCAGC } \\
\text { TTCCTTACACTTCCCAAGAGGAGAA } \\
\text { GCAGTTTGGAAAAACAAAATCAGA } \\
\text { ATAAGTTGGTCCTGAGTTCTAACTT } \\
\text { TGGCTCTTCACCTTTCTAGTCCCCAA } \\
\text { TTTATATTGTTCCTCCGTGCGTCAGT } \\
\text { TTTACCTGTGAGATAAGGCCAGTAG } \\
\text { CCAGCCCCGTCCTGGCAGGGCTGTG } \\
\text { GTGAGGAGGGGGGTGTCCGTGTGG } \\
\text { AAAACTCCCTTTGTGAGAATGGTGC } \\
\text { GTCCTAGGTGTTCACCAGGTCGTGG } \\
\text { CCGCCTCTACTCCCTTCTCTTTCTC } \\
\text { CATCCTTCTTTCCTTAAAGAGTCCCC } \\
\text { AGTGCTATCTGGGACATATTCCTCC } \\
\text { GCCCAGAGCAGGGTCCCGCTTCCCT } \\
\text { AAGGCCCTGCTCTGGGCTTCTGGGT } \\
\text { TTGAGTCCTTGGCAAGCCCAGGAGA } \\
\text { GGCGCTCAGGCTTCCCTGTCCCCCT } \\
\text { TCCTCGTCCACCATCTCATGCCCCT } \\
\text { GGCTCTCCTGCCCCTTCCCTACAGG } \\
\text { GGTTCCT }\end{array}$ \\
\hline $\mathrm{CCR} 5^{7}$ & $\begin{array}{c}\text { GGAGAGCT } \\
\text { TGGCTCTGT } \\
\text { TGGGGG }\end{array}$ & $\begin{array}{l}\text { CCGGCCATTTCACTCTGACTACATC } \\
\text { ATGTCACCAAACATCTGATGGTCTT } \\
\text { GCCTTTTAATTCTCTTTTCGAGGACT } \\
\text { GAGAGGGAGGGTAGCATGGTAGTT } \\
\text { AAGAGTGCAGGCTTCCCGCATTCAA } \\
\text { AATCGGTTGCTTACTAGCTGTGTGG } \\
\text { CTTTGAGCAAGTTACTCACCCTCTC } \\
\text { TGTGCTTCAAGGTCCTTGTCTGCAA } \\
\text { AATGTGAAAAATATTTCCTGCCTCA } \\
\text { TAAGGTTGCCCTAAGGATTAAATGA } \\
\text { ATGAATGGGTATGATGCTTAGAACA } \\
\text { GTGATTGGCATCCAGTATGTGCCCT } \\
\text { CGAGGCCTCTTAATTATTACTGGCT } \\
\text { TGCTCATAGTGCATGTTCTTTGTGG } \\
\end{array}$ & $\begin{array}{l}\text { ACAGAGCCAAGCTCTCCATCTAGTG } \\
\text { GACAGGGAAGCTAGCAGCAAACCT } \\
\text { TCCCTTCACTACAAAACTTCATTGC } \\
\text { TTGGCCAAAAAGAGAGTTAATTCAA } \\
\text { TGTAGACATCTATGTAGGCAATTAA } \\
\text { AAACCTATTGATGTATAAAACAGTT } \\
\text { TGCATTCATGGAGGGCAACTAAATA } \\
\text { CATTCTAGGACTTTATAAAAGATCA } \\
\text { CTTTTTATTTATGCACAGGGTGGAA } \\
\text { CAAGATGGATTATCAAGTGTCAAGT } \\
\text { CCAATCTATGACATCAATTATTATA } \\
\text { CATCGGAGCCCTGCCAAAAAATCA } \\
\text { ATGTGAAGCAAATCGCAGCCCGCCT } \\
\text { CCTGCCTCCGCTCTACTCACTGGTG }\end{array}$ \\
\hline
\end{tabular}




\begin{tabular}{|c|c|c|c|}
\hline & & $\begin{array}{l}\text { GCTAACTCTAGCGTCAATAAAAATG } \\
\text { TTAAGACTGAGTTGCAGCCGGGCAT } \\
\text { GGTGGCTCATGCCTGTAATCCCAGC } \\
\text { ATTCTAGGAGGCTGAGGCAGGAGG } \\
\text { ATCGCTTGAGCCCAGGAGTTCGAGA } \\
\text { CCAGCCTGGGCAACATAGTGTGATC } \\
\text { TTGTATCTATAAAAATAAACAAAAT } \\
\text { TAGCTTGGTGTGGTGGCGCCTGTAG } \\
\text { TCCCCAGCCACTTGGAGGGGTGAGG } \\
\text { TGAGAGGATTGCTTGAGCCCGGGAT } \\
\text { GGTCCAGGCTGCAGTGAGCCATGAT } \\
\text { CGTGCCACTGCACTCCAGCCTGGGC } \\
\text { GACAGAGTGAGACCCTGTCTCACAA } \\
\text { CAACAACAACAACAACAAAAAGGC } \\
\text { TGAGCTGCACCATGCTTGACCCAGT } \\
\text { TTCTTAAAATTGTTGTCAAAGCTTC } \\
\text { ATTCACTCCATGGTGCTATAGAGCA } \\
\text { CAAGATTTTATTTGGTGAGATGGTG } \\
\text { CTTTCATGAATTCCCCA }\end{array}$ & $\begin{array}{l}\text { TTCATCTTTGGTTTTGTGGGCAACAT } \\
\text { GCTGGTCATCCTCATCCTGATAAAC } \\
\text { TGCAAAAGGCTGAAGAGCATGACT } \\
\text { GACATCTACCTGCTCAACCTGGCCA } \\
\text { TCTCTGACCTGTTTTCCTTCTTACT } \\
\text { GTCCCTTCTGGGCTCACTATGCTG } \\
\text { CCGCCCAGTGGGACTTTGGAAATAC } \\
\text { AATGTGTCAACTCTTGACAGGGCTC } \\
\text { TATTTTATAGGCTTCTTCTCTGGAAT } \\
\text { CTTCTTCATCATCCTCCTGACAATCG } \\
\text { ATAGGTACCTGGCTGTCGTCCATGC } \\
\text { TGTGTTTGCTTTAAAAGCCAGGACG } \\
\text { GTCACCTTTGGGGTGGTGACAAGTG } \\
\text { TGATCACTTGGGTGGTGGCTGTGTT } \\
\text { TGCGTCTCTCCCAGGAATCATCTTT } \\
\text { ACCAGATCTCAAAAAGAAGGTCTTC } \\
\text { ATTACACCTGCAGCTCTCATTTTCC } \\
\text { ATACAGTCAGTATCAATTCTGGAAG } \\
\text { AATTTCCAGACATTAAAGATAGTCA } \\
\text { TCTTGGGGCTGGTCCTGCCGCTGCT } \\
\text { TGTCATGGTCATCTGCTACTCGGGA } \\
\text { A }\end{array}$ \\
\hline ROSA $26^{8}$ & $\begin{array}{c}\text { GTCGAGTC } \\
\text { GCTTCTCGA } \\
\text { TTATGG }\end{array}$ & $\begin{array}{l}\text { AAGCTCCTGTCAGTTACGCCGTCGG } \\
\text { GAGTACGCAGCCGCTTAGCGACTCT } \\
\text { CGCGTTGCCCCCTGGGTGGGGCGGG } \\
\text { TAGGTAGGTGGGGTGTAGAGATGCT } \\
\text { GGGTGTGCGGGCGCGGCCGGCTCC } \\
\text { TGCGGCGGGAGGGAGGTCAGTG } \\
\text { AAATCGGCTCTGGCGCGGGCGTCCT } \\
\text { CCCACCCTCCCCTCCTTCGGGGGA } \\
\text { GTCGGTTTACCCGCCGCCTGCTTGT } \\
\text { CTTCGACACCTGATTGGCTGTCGAA } \\
\text { GCTGTGGGACCGGGCCCTTGCTACT } \\
\text { GGCTCGAGTCTCACATGAGCGAAAC } \\
\text { CACTGCGCGGGGCGCGGGGGTGGC } \\
\text { GGGGAGGCGGGCGTTGGTACGGTC } \\
\text { CTCCCCGAGGCCGAGCGCCGCAGTG } \\
\text { TCTGGCCCCGCGCCCCTGCGCAACG } \\
\text { TGGCAGGAAGCGCGCGCTGGAGGC } \\
\text { GGGGGCGGGCTGCCGGCGAGACT } \\
\text { TCTGGATGGCGGCGGCCGCGGCTCC } \\
\text { GCCCCGGGTTCCACCGCCTGAAGG } \\
\text { GCGAGACAAGCCCGACCTGCTACA } \\
\text { GGCACTCGTGGGGGTGGGGGAGGA } \\
\text { GCGGGGGTCGGTCCGGCTGGTTTGT } \\
\text { GGGTGGGAGGCGCTTGTTCTCCAAA } \\
\text { AACCGGCGCGAGCTGCAATCCTGA } \\
\text { GGGAGCTGCGGTGGAGGAGGTGGA } \\
\text { GAGAAGGCCGCACCCTTCTGGGCA } \\
\text { GGGGGAGGGGAGTGCCGCAATACC } \\
\text { TTTATGGGAGTTCTCTGCTGCCTCCC } \\
\text { GTCTTGTAAGGACCGCCCTGGGCCT } \\
\text { GGAAGAAGCCCTCCTCCTTCCTC } \\
\text { CTCGCGTGATCTCGTCATCGCCTCC } \\
\text { ATGTCGAGTCGCTTCTCGA }\end{array}$ & $\begin{array}{l}\text { TTATGGGCGGGATTCTTTTGCCTAG } \\
\text { GCTTAAGGGGCTAACTTGGTCCCTG } \\
\text { GGCGTTGCCCTGCAGGGGAGTGAG } \\
\text { CAGCTGTAAGATTTGAGGGGCGACT } \\
\text { CCGATTAGTTTATCTTCCCACGGAC } \\
\text { TAGAGTTGGTGTCGAGGTATTGTA } \\
\text { ATAAGGGTGGGTAGGGAAATGGA } \\
\text { GCTTAGTCATTCACCTGGGGCTGAT } \\
\text { TTTATGCAACGAGACTGCGGATTAT } \\
\text { CACTACTTATCATTTTTGGAGCATTT } \\
\text { TTCTAGAGACAGACATAAAGCATG } \\
\text { ATCACCTGAGTTTATACCATTTGA } \\
\text { GACCCTTGCTGCACCACCAAAGTGT } \\
\text { AGCATCAGGTTAAATCTTAATAGAA } \\
\text { AAATTTTAGCTTTTGCTTGAGAAAC } \\
\text { CAGTGCTTCCCTCCCTCACCCTCTCT } \\
\text { CCCCAGGCTCTCTACCCCTTTGCAT } \\
\text { CCCTACCAGGCATCTTAGCAACTCT } \\
\text { CACTCATACTTGATCCCATTTTCCAT } \\
\text { TTGTTGTACTTGCTCCTCTAGTATTC } \\
\text { AGACATAGCACTAGCTTTCTCCCTC } \\
\text { TCTTGATCTTGGGTAGCCTGGTGTC } \\
\text { TCGCGAAACCAGACAGATTGGTTCC } \\
\text { ACCACAAATTAAGGCTTGAGCTGGG } \\
\text { GCTTGACTCTTACCCAGCAGTGCTT } \\
\text { TTATTCCTCCCTAGTTCACGTTCTTA } \\
\text { AATGTTTATCTTGATTTTCATTTTAT } \\
\text { CCTTTTTCCTTAGCTGGGATTCTGTC } \\
\text { CCTGACCGTCTTCACAGTCCAGGTG } \\
\text { ATCTTGACTACTGCTTTACAGAGAA } \\
\text { TTGGATCTGAGGTTAGGCAACATCT } \\
\text { CCCTTTTTCTTCCTCTAAATACCTCT } \\
\text { CATTTCTGTTCTTACCAGTTAGTAAC } \\
\text { TGATCTCAGATGCCTGTGTGATAGC } \\
\text { TTCCAA }\end{array}$ \\
\hline
\end{tabular}




\begin{tabular}{|c|c|c|c|}
\hline \multirow{2}{*}{$\begin{array}{c}\mathrm{CMV} \\
\text { promoter }\end{array}$} & $\begin{array}{l}\text { TTACCGTA } \\
\text { AGTTATGT } \\
\text { AACGCGG } \\
\end{array}$ & $\mathrm{N} / \mathrm{A}$ & $\mathrm{N} / \mathrm{A}$ \\
\hline & $\begin{array}{c}\text { GTGGCGAC } \\
\text { CGGTAGCG } \\
\text { CTAGCGG }\end{array}$ & $\mathrm{N} / \mathrm{A}$ & N/A \\
\hline $\begin{array}{l}\text { CMV } \\
\text { D.C. }\end{array}$ & $\begin{array}{l}\text { TGGAGTTC } \\
\text { CGCGTGCG } \\
\text { CTACCGG }\end{array}$ & $\begin{array}{l}\text { AAGGTGTGGGTCGCGGACGACGGC } \\
\text { GCCGCGGTGGCGGTCTGGACCACGC } \\
\text { CGGAGAGCGTCGAAGCGGGGGCGG } \\
\text { TGTTCGCCGAGATCGGCCCGCGCAT } \\
\text { GGCCGAGTTGAGCGGTCCCGGCTG } \\
\text { GCCGCGCAGCAACAGATGGAAGGC } \\
\text { CTCCTGGCGCCGCACCGGCCCAAGG } \\
\text { AGCCCGCGTGGTCCTGGCCACCGT } \\
\text { CGGCGTCTCGCCCGACCACCAGGGC } \\
\text { AAGGGTCTGGGCAGCGCCGTCGTGC } \\
\text { TCCCCGGAGTGGAGGCGGCCGAGC } \\
\text { GCGCCGGGGTGCCCGCCTTCCTGGA } \\
\text { GACCTCCGCGCCCCGCAACCTCCC } \\
\text { TTCTACGAGCGGCTCGGCTTCACCG } \\
\text { TCACCGCCGACGTCGAGGTGCCCGA } \\
\text { AGGACCGCGCACCTGGTGCATGACC } \\
\text { CGCAAGCCCGGTGCCTGATCTAGAG } \\
\text { GGCCCGTTTAAACCCGCTGATCAGC } \\
\text { CTCGACTGTGCCTTCTAGTTGCCAG } \\
\text { CCATCTGTTGTTTGCCCTCCCCGT } \\
\text { GCCTTCCTTGACCCTGGAAGGTGCC } \\
\text { ACTCCCACTGTCCTTTCCTAATAAA } \\
\text { ATGAGGAAATTGCATCGCATTGTCT } \\
\text { GAGTAGGTGTCATTCTATTCTGGGG } \\
\text { GGTGGGGTGGGGCAGGACAGCAAG } \\
\text { GGGGAGGATTGGGAAGACAATAGC } \\
\text { AGGCATGCTGGGGATGCGGTGGGC } \\
\text { TCTATGGAAAGCCTATTCTTTCCTG } \\
\text { CGTTATCCCCTGATTCTGTGGATAA } \\
\text { CCGTATTACCGCCATGCATTAGTTA } \\
\text { TTAATAGTAATCAATTACGGGGTCA } \\
\text { TTAGTTCATAGCCCATATA }\end{array}$ & $\begin{array}{l}\text { CGCCACCATGGTGAGCAAGGGCGA } \\
\text { GGAGCTGTTCACCGGGGTGGTGCCC } \\
\text { ATCCTGGTCGAGCTGGACGGCGACG } \\
\text { TAAACGGCCACAAGTTCAGCGTGTC } \\
\text { CGGCGAGGGCGAGGGCGATGCCAC } \\
\text { CTACGGCAAGCTGACCCTGAAGTTC } \\
\text { ATCTGCACCACCGGCAAGCTGCCCG } \\
\text { TGCCCTGGCCCACCCTCGTGACCAC } \\
\text { CCTGACCTACGGCGTGCAGTGCTTC } \\
\text { AGCCGCTACCCCGACCACATGAAGC } \\
\text { AGCACGACTTCTTCAAGTCCGCCAT } \\
\text { GCCCGAAGGTACGTCCAGGAGCG } \\
\text { CACCATCTTCTTCAAGGACGACGGC } \\
\text { AACTACAAGACCCGCGCCGAGGTG } \\
\text { AAGTTCGAGGGCGACACCCTGGTG } \\
\text { AACCGCATCGAGCTGAAGGGCATC } \\
\text { GACTTCAAGGAGGACGGCAACATC } \\
\text { CTGGGGCACAAGCTGGAGTACAAC } \\
\text { TACAACAGCCACAACGTCTATATCA } \\
\text { TGGCCGACAAGCAGAAGAACGGCA } \\
\text { TCAAGGTGAACTTCAAGATCCGCCA } \\
\text { CAACATCGAGGACGGCAGCGTGCA } \\
\text { GCTCGCCGACCACTACCAGCAGAAC } \\
\text { ACCCCATCGGCGACGGCCCGTGC } \\
\text { TGCTGCCCGACAACCACTACCTGAG } \\
\text { CACCCAGTCCGCCCTGAGCAAAGAC } \\
\text { CCCAACGAGAAGCGCGATCACATG } \\
\text { GTCCTGCTGGAGTTCGTGACCGCCG } \\
\text { CCGGGATCACTCTCGGCATGGACGA } \\
\text { GCTGTACAAGTCCGGACTCAGATCT } \\
\text { CGAGCTCAAGC }\end{array}$ \\
\hline
\end{tabular}


Supporting Table S2. Plasmid information.

\begin{tabular}{|c|c|}
\hline Plasmid name & Description \\
\hline AAVS1-sgRNA-Cas9 & sgRNA targeting AAVS1 locus and Cas9 expression vector \\
\hline CCR5-sgRNA-Cas9 & sgRNA targeting CCR5 locus and Cas9 expression vector \\
\hline ROSA26-sgRNA-Cas9 & sgRNA targeting ROSA26 locus and Cas9 expression vector \\
\hline AAVS1-eGFP donor & Donor plasmid targeting AAVS1 locus (GOI: eGFP) \\
\hline CCR5-eGFP donor & Donor plasmid targeting CCR5 locus (GOI: eGFP) \\
\hline ROSA26-eGFP donor & Donor plasmid targeting ROSA26 locus (GOI: eGFP) \\
\hline CMV-sgRNA1-Cas9 & sgRNA targeting CMV promoter site 1 and Cas9 expression vector \\
\hline CMV-sgRNA3-Cas9 & sgRNA targeting CMV promoter site 3 and Cas9 expression vector \\
\hline CMV-D.C.-sgRNA-Cas9 & sgRNA targeting CMV-D.C. site and Cas9 expression vector \\
\hline CMV-D.C.-EF1 $\alpha$ donor & Donor plasmid targeting CMV-D.C. site (GOI: EF1 $\alpha$ promoter) \\
\hline CMV-D.C.-SV40 donor & Donor plasmid targeting CMV-D.C. site (GOI: SV40 promoter) \\
\hline CMV-D.C.-TK donor & Donor plasmid targeting CMV-D.C. site (GOI: TK promoter) \\
\hline CMV-D.C.-CMV donor & Donor plasmid targeting CMV-D.C. site (GOI: CMV promoter) \\
\hline AAVS1-LP donor & Donor plasmid targeting CMV locus (GOI: LP) \\
\hline RMCE-CMV-ETN donor & Donor plasmid for RMCE with LP (GOI: CMV-ETN) \\
\hline RMCE-EF1 $1 \alpha-E T N$ donor & Donor plasmid for RMCE with LP (GOI: EF1 $\alpha$-ETN) \\
\hline RMCE-CMV-EPO donor & Donor plasmid for RMCE with LP (GOI: CMV-EPO) \\
\hline RMCE-EF1 $\alpha$-EPO donor & Donor plasmid for RMCE with LP (GOI: EF1 $\alpha$-EPO) \\
\hline PSF-CMV-CRE & Cre recombinase expression vector from Sigma-Aldrich \\
\hline
\end{tabular}




\begin{tabular}{|c|c|}
\hline $\begin{array}{l}\text { Plasmid name } \\
\text { (PCR template) }\end{array}$ & Element \\
\hline $\begin{array}{c}\text { pU6-(BbsI)_CBh-Cas9- } \\
\text { T2A-mCherry }\end{array}$ & Backbone of sgRNA-Cas9 vectors (Addgene plasmid \#64324) ${ }^{6}$ \\
\hline $\begin{array}{l}\text { pAAVS1-TLR targeting } \\
\text { vector }\end{array}$ & $\begin{array}{l}\text { Splicing acceptor, T2A, and puromycin resistance gene (Addgene } \\
\text { plasmid } \# 64215)^{6}\end{array}$ \\
\hline pEGFP-C1 & eGFP gene, CMV promoter (Clontech) \\
\hline $\begin{array}{l}\text { pAAVS1-TLR targeting } \\
\text { vector }\end{array}$ & Backbone of eGFP donor plasmids (Addgene plasmid \#64215) ${ }^{6}$ \\
\hline $\begin{array}{l}\text { COSMC-mCherry-HDR-TI } \\
\text { donor }\end{array}$ & EF1 $\alpha$ promoter $^{1}$ \\
\hline pcDNA3.1/Zeo $(+)$ & SV40 promoter (Life Technologies) \\
\hline pCEP4 & TK donor promoter (Life Technologies) \\
\hline $\operatorname{pcDNA3.1}(+)$ & Backbone of CMV-D.C.-promoter donor plasmids (Life Technologies) \\
\hline LP donor plasmid & LP (loxP-EF1 $\alpha$-mCherry-lox2272-BGH poly(A)) sequence ${ }^{5}$ \\
\hline pcDNA3.1(+)-Enbrel & ETN gene \\
\hline RMCE EPO donor plasmid & EPO gene, lox2272-backbone-loxP sequence ${ }^{5}$ \\
\hline
\end{tabular}


Supporting Table S3. Primer sequences.

\begin{tabular}{|c|c|c|}
\hline Primer name & Description & Sequence (5'-3') \\
\hline AAVS1_sgRNA_fwd & $\begin{array}{l}\text { Sense oligo for AAVS1- } \\
\text { sgRNA }\end{array}$ & $\begin{array}{l}\text { CACCGACCCCACAGTGGG } \\
\text { GCCACTA }\end{array}$ \\
\hline AAVS1_sgRNA_rev & $\begin{array}{l}\text { Antisense oligo for AAVS1- } \\
\text { sgRNA }\end{array}$ & $\begin{array}{l}\text { AAACTAGTGGCCCCACTGT } \\
\text { GGGGTC }\end{array}$ \\
\hline CCR5_sgRNA_fwd & $\begin{array}{l}\text { Sense oligo for CCR5- } \\
\text { sgRNA }\end{array}$ & $\begin{array}{l}\text { CACCGGGAGAGCTTGGCTC } \\
\text { TGTTGG }\end{array}$ \\
\hline CCR5_sgRNA_rev & $\begin{array}{l}\text { Antisense oligo for CCR5- } \\
\text { sgRNA }\end{array}$ & $\begin{array}{l}\text { AAACCCAACAGAGCCAAG } \\
\text { CTCTCCC }\end{array}$ \\
\hline ROSA26_sgRNA_fwd & $\begin{array}{l}\text { Sense oligo for ROSA26- } \\
\text { sgRNA }\end{array}$ & $\begin{array}{l}\text { CACCGGTCGAGTCGCTTCT } \\
\text { CGATTA }\end{array}$ \\
\hline ROSA26_sgRNA_rev & $\begin{array}{l}\text { Antisense oligo for } \\
\text { ROSA26-sgRNA }\end{array}$ & $\begin{array}{l}\text { AAACTAATCGAGAAGCGA } \\
\text { CTCGACC }\end{array}$ \\
\hline AAVS1_5'arm_fwd & $\begin{array}{l}\text { USER primer for AAVS1- } \\
\text { eGFP donor plasmid }\end{array}$ & $\begin{array}{l}\text { AGTCGGTGUCCGTCTCTCT } \\
\text { CCTGAGTCCG }\end{array}$ \\
\hline AAVS1_5'arm_rev & $\begin{array}{l}\text { USER primer for AAVS1- } \\
\text { eGFP donor plasmid }\end{array}$ & $\begin{array}{l}\text { ACGCTGCTUACTGTGGGGT } \\
\text { GGAGGGGACAGAT }\end{array}$ \\
\hline AAVS1_3'arm_fwd & $\begin{array}{l}\text { USER primer for AAVS1- } \\
\text { eGFP donor plasmid }\end{array}$ & $\begin{array}{l}\text { AGACGTCAUGGGGCCACT } \\
\text { AGGGACAGGAT }\end{array}$ \\
\hline AAVS1_3'arm_rev & $\begin{array}{l}\text { USER primer for AAVS1- } \\
\text { eGFP donor plasmid }\end{array}$ & $\begin{array}{l}\text { ACGCAAGUAGGAACCCCT } \\
\text { GTAGGGAAGG }\end{array}$ \\
\hline CCR5_5'arm_fwd & $\begin{array}{l}\text { USER primer for CCR5- } \\
\text { eGFP donor plasmid }\end{array}$ & $\begin{array}{l}\text { AGTCGGTGUCCGGCCATTT } \\
\text { CACTCTGACTA }\end{array}$ \\
\hline CCR5_5'arm_rev & $\begin{array}{l}\text { USER primer for CCR5- } \\
\text { eGFP donor plasmid }\end{array}$ & $\begin{array}{l}\text { ACGCTGCTUTGGGGGAATT } \\
\text { CATGAAAGCA }\end{array}$ \\
\hline CCR5_3'arm_fwd & $\begin{array}{l}\text { USER primer for CCR5- } \\
\text { eGFP donor plasmid }\end{array}$ & $\begin{array}{l}\text { AGACGTCAUACAGAGCCA } \\
\text { AGCTCTCCATCT }\end{array}$ \\
\hline CCR5_3'arm_rev & $\begin{array}{l}\text { USER primer for CCR5- } \\
\text { eGFP donor plasmid }\end{array}$ & $\begin{array}{l}\text { ACGCAAGUTTCCCGAGTA } \\
\text { GCAGATGACC }\end{array}$ \\
\hline ROSA26_5'arm_fwd & $\begin{array}{l}\text { USER primer for ROSA26- } \\
\text { eGFP donor plasmid }\end{array}$ & $\begin{array}{l}\text { AGTCGGTGUAAGCTCCTGT } \\
\text { CAGTTACGCC }\end{array}$ \\
\hline ROSA26_5'arm_rev & $\begin{array}{l}\text { USER primer for ROSA26- } \\
\text { eGFP donor plasmid }\end{array}$ & $\begin{array}{l}\text { ACGCTGCTUTCGAGAAGC } \\
\text { GACTCGACATGG }\end{array}$ \\
\hline ROSA26_3'arm_fwd & $\begin{array}{l}\text { USER primer for ROSA26- } \\
\text { eGFP donor plasmid }\end{array}$ & $\begin{array}{l}\text { AGACGTCAUTTATGGGCG } \\
\text { GGATTCTTTTG }\end{array}$ \\
\hline ROSA26_3'arm_rev & $\begin{array}{l}\text { USER primer for ROSA26- } \\
\text { eGFP donor plasmid }\end{array}$ & $\begin{array}{l}\text { ACGCAAGUTTGGAAGCTA } \\
\text { TCACACAGGC }\end{array}$ \\
\hline SA_T2A_PuroR_fwd & $\begin{array}{l}\text { USER primer for eGFP } \\
\text { donor plasmid }\end{array}$ & $\begin{array}{l}\text { AAGCAGCGUAAGCTTCTG } \\
\text { ACCTCTTCTC }\end{array}$ \\
\hline SA_T2A_PuroR_rev & $\begin{array}{c}\text { USER primer for eGFP } \\
\text { donor plasmid }\end{array}$ & $\begin{array}{l}\text { ATAGGCTTUCCATAGAGCC } \\
\text { CACCGCATC }\end{array}$ \\
\hline CMV_eGFP_fwd & $\begin{array}{l}\text { USER primer for eGFP } \\
\text { donor plasmid }\end{array}$ & $\begin{array}{l}\text { AAAGCCTAUTCTTTCCTGC } \\
\text { GTTATCCCCTG }\end{array}$ \\
\hline
\end{tabular}




\begin{tabular}{|c|c|c|}
\hline CMV_eGFP_rev & $\begin{array}{l}\text { USER primer for eGFP } \\
\text { donor plasmid }\end{array}$ & $\begin{array}{l}\text { ATGACGTCUCGCGTTAAGA } \\
\text { TACATTGATGAG }\end{array}$ \\
\hline pAAVS1_TLR_backbone_fwd & $\begin{array}{l}\text { USER primer for eGFP } \\
\text { donor plasmid }\end{array}$ & $\begin{array}{l}\text { ACTTGCGUAAGGGCGGCC } \\
\text { GCTAAATTC }\end{array}$ \\
\hline pAAVS1_TLR_backbone_rev & $\begin{array}{l}\text { USER primer for eGFP } \\
\text { donor plasmid }\end{array}$ & $\begin{array}{l}\text { ACACCGACUAAGGGCGAA } \\
\text { TTCGTTTAAACCTG }\end{array}$ \\
\hline AAVS1_5'junction_fwd & $\begin{array}{l}\text { AAVS1 amplicon for 5' } \\
\text { junction PCR }\end{array}$ & $\begin{array}{l}\text { GGCAGCCTGTGCTGACCCA } \\
\text { TGCAGTC }\end{array}$ \\
\hline AAVS1_5'junction_rev & $\begin{array}{l}\text { AAVS1 amplicon for 5' } \\
\text { junction PCR }\end{array}$ & $\begin{array}{l}\text { CGGGATTCTCCTCCACGTC } \\
\text { ACCGCA }\end{array}$ \\
\hline AAVS1_3'junction_fwd & $\begin{array}{l}\text { AAVS1 amplicon for 3' } \\
\text { junction PCR }\end{array}$ & $\begin{array}{l}\text { AGATCCGCCACAACATCG } \\
\text { AG }\end{array}$ \\
\hline AAVS1_3'junction_rev & $\begin{array}{l}\text { AAVS1 amplicon for 3' } \\
\text { junction PCR }\end{array}$ & $\begin{array}{l}\text { GCAATTGGGGTGCAGGAA } \\
\text { TG }\end{array}$ \\
\hline CCR5_5'junction_fwd & $\begin{array}{l}\text { CCR5 amplicon for 5' } \\
\text { junction PCR }\end{array}$ & $\begin{array}{l}\text { TTCATCTGTGGTGGCAGAC } \\
\text { G }\end{array}$ \\
\hline CCR5_5'junction_rev & $\begin{array}{l}\text { CCR5 amplicon for 5 } \\
\text { junction PCR }\end{array}$ & $\begin{array}{l}\text { CGGGATTCTCCTCCACGTC } \\
\text { ACCGCA }\end{array}$ \\
\hline CCR5_3'junction_fwd & $\begin{array}{l}\text { CCR5 amplicon for 3' } \\
\text { junction PCR }\end{array}$ & $\begin{array}{l}\text { GACTCAGATCTCGAGCTCA } \\
\text { AGCTTCG }\end{array}$ \\
\hline CCR5_3'junction_rev & $\begin{array}{l}\text { CCR5 amplicon for 3' } \\
\text { junction PCR }\end{array}$ & $\begin{array}{l}\text { TTGATGCAGCAGTGCGTCA } \\
\text { TCCCAAG }\end{array}$ \\
\hline ROSA26_5'junction_fwd & $\begin{array}{l}\text { ROSA26 amplicon for 5' } \\
\text { junction PCR }\end{array}$ & $\begin{array}{l}\text { CTAGCCTCTTGTCGCCGAT } \\
\text { T }\end{array}$ \\
\hline ROSA26_5'junction_rev & $\begin{array}{l}\text { ROSA26 amplicon for 5' } \\
\text { junction PCR }\end{array}$ & $\begin{array}{l}\text { ACTCGGTCATCTCGAGCCT } \\
\text { A }\end{array}$ \\
\hline ROSA26_3'junction_fwd & $\begin{array}{l}\text { ROSA26 amplicon for 3' } \\
\text { junction PCR }\end{array}$ & $\begin{array}{l}\text { GACTCAGATCTCGAGCTCA } \\
\text { AGCTTCG }\end{array}$ \\
\hline ROSA26_3'junction_rev & $\begin{array}{l}\text { ROSA26 amplicon for 3' } \\
\text { junction PCR }\end{array}$ & $\begin{array}{l}\text { GTTGGGTCGCATTCAAAGC } \\
\text { TGTCCTG }\end{array}$ \\
\hline eGFP_fwd & $\begin{array}{l}\text { eGFP amplicon for qRT- } \\
\text { PCR }\end{array}$ & GAACCGCATCGAGCTGAA \\
\hline eGFP_rev & $\begin{array}{l}\text { eGFP amplicon for qRT- } \\
\text { PCR }\end{array}$ & $\begin{array}{l}\text { TGCTTGTCGGCCATGATAT } \\
\text { AG }\end{array}$ \\
\hline $\mathrm{ACTB}_{-}$fwd & $\begin{array}{l}\text { ACTB amplicon for qRT- } \\
\text { PCR }\end{array}$ & $\begin{array}{l}\text { CTGGAACGGTGAAGGTGA } \\
\text { CA }\end{array}$ \\
\hline ACTB_rev & $\begin{array}{l}\text { ACTB amplicon for qRT- } \\
\text { PCR }\end{array}$ & $\begin{array}{l}\text { AAGGGACTTCCTGTAACAA } \\
\text { CGCA }\end{array}$ \\
\hline CMV_sgRNA_1_fwd & $\begin{array}{l}\text { Sense oligo for CMV- } \\
\text { sgRNA1 }\end{array}$ & $\begin{array}{l}\text { GGAAAGGACGAAACACCG } \\
\text { TACCGTAAGTTATGTAACG } \\
\text { GTTTTAGAGCTAGAAAT }\end{array}$ \\
\hline CMV_sgRNA_1_rev & $\begin{array}{l}\text { Antisense oligo for CMV- } \\
\text { sgRNA1 }\end{array}$ & $\begin{array}{l}\text { CTAAAACCGTTACATAACT } \\
\text { TACGGTACGGTGTTTCGTC } \\
\text { CTTTCCACAAGATAT }\end{array}$ \\
\hline
\end{tabular}




\begin{tabular}{|c|c|c|}
\hline CMV_sgRNA_3_fwd & $\begin{array}{l}\text { Sense oligo for CMV- } \\
\text { sgRNA3 }\end{array}$ & $\begin{array}{l}\text { GGAAAGGACGAAACACCG } \\
\text { TGGCGACCGGTAGCGCTA } \\
\text { GGTTTTAGAGCTAGAAAT }\end{array}$ \\
\hline CMV_sgRNA_3_rev & $\begin{array}{l}\text { Antisense oligo for CMV- } \\
\text { sgRNA3 }\end{array}$ & $\begin{array}{l}\text { CTAAAACCTAGCGCTACCG } \\
\text { GTCGCCACGGTGTTTCGTC } \\
\text { CTTTCCACAAGATAT }\end{array}$ \\
\hline CMV_D.C._fwd & $\begin{array}{l}\text { CMV-D.C. amplicon for } \\
\text { PCR }\end{array}$ & $\begin{array}{l}\text { CGGAACTCTGCCCTCTAAC } \\
\text { G }\end{array}$ \\
\hline CMV_D.C._rev & $\begin{array}{l}\text { CMV-D.C. amplicon for } \\
\text { PCR }\end{array}$ & GAACAGCTCCTCGCCCTTG \\
\hline CMV_D.C._seq_fwd & $\begin{array}{l}\text { CMV-D.C. amplicon } \\
\text { sequencing }\end{array}$ & $\begin{array}{l}\text { CATGGCCGAGTTGAGCGGT } \\
\mathrm{T}\end{array}$ \\
\hline CMV_D.C._sgRNA_fwd & $\begin{array}{l}\text { Sense oligo for CMV-D.C.- } \\
\text { sgRNA1 }\end{array}$ & $\begin{array}{l}\text { CACCGTGGAGTTCCGCGTG } \\
\text { CGCTAC }\end{array}$ \\
\hline CMV_D.C._sgRNA_rev & $\begin{array}{l}\text { Antisense oligo for CMV- } \\
\text { D.C.-sgRNA1 }\end{array}$ & $\begin{array}{l}\text { AAACGTAGCGCACGCGGA } \\
\text { ACTCCAC }\end{array}$ \\
\hline CMV_D.C._5'arm_fwd & $\begin{array}{l}\text { USER primer for promoter } \\
\text { donor plasmid }\end{array}$ & $\begin{array}{l}\text { AGTCGGTGUAAGGTGTGG } \\
\text { GTCGCGGAC }\end{array}$ \\
\hline CMV_D.C._5'arm_rev & $\begin{array}{l}\text { USER primer for promoter } \\
\text { donor plasmid }\end{array}$ & $\begin{array}{l}\text { ACGCTGCTUTATATGGGCT } \\
\text { ATGAACTAATGACC }\end{array}$ \\
\hline CMV_D.C._3'arm_fwd & $\begin{array}{l}\text { USER primer for promoter } \\
\text { donor plasmid }\end{array}$ & $\begin{array}{l}\text { AGACGTCAUCGCCACCAT } \\
\text { GGTGAGCAAGG }\end{array}$ \\
\hline CMV_D.C._3'arm_rev & $\begin{array}{l}\text { USER primer for promoter } \\
\text { donor plasmid }\end{array}$ & $\begin{array}{l}\text { ACGCAAGUGCTTGAGCTC } \\
\text { GAGATCTGAG }\end{array}$ \\
\hline EF1 $\alpha \_f w d$ & $\begin{array}{l}\text { USER primer for EF1 } \alpha \\
\text { donor plasmid }\end{array}$ & $\begin{array}{l}\text { AAGCAGCGUGTGAGGCTC } \\
\text { CGGTGCCCGT }\end{array}$ \\
\hline EF1a_rev & $\begin{array}{l}\text { USER primer for EF1 } \alpha \\
\text { donor plasmid }\end{array}$ & $\begin{array}{l}\text { ATGACGTCUTCACGACACC } \\
\text { TGAAATGGAAGA }\end{array}$ \\
\hline SV40_fwd & $\begin{array}{c}\text { USER primer for SV40 } \\
\text { donor plasmid }\end{array}$ & $\begin{array}{l}\text { AAGCAGCGUGTGTGTCAG } \\
\text { TTAGGGTGTGGA }\end{array}$ \\
\hline SV40_rev & $\begin{array}{l}\text { USER primer for SV40 } \\
\text { donor plasmid }\end{array}$ & $\begin{array}{l}\text { ATGACGTCUGTGCTGATCA } \\
\text { GATCCGAAAATGG }\end{array}$ \\
\hline TK_fwd & $\begin{array}{l}\text { USER primer for TK donor } \\
\text { plasmid }\end{array}$ & $\begin{array}{l}\text { AAGCAGCGUATGACACAA } \\
\text { ACCCCGCCC }\end{array}$ \\
\hline TK_rev & $\begin{array}{l}\text { USER primer for TK donor } \\
\text { plasmid }\end{array}$ & $\begin{array}{l}\text { ATGACGTCUTTAAGCGGGT } \\
\text { CGCTGCAGG }\end{array}$ \\
\hline CMV_fwd & $\begin{array}{l}\text { USER primer for CMV } \\
\text { donor plasmid }\end{array}$ & $\begin{array}{l}\text { AAGCAGCGUATAGTAATC } \\
\text { AATTACGGGGTCA }\end{array}$ \\
\hline CMV_rev & $\begin{array}{l}\text { USER primer for CMV } \\
\text { donor plasmid }\end{array}$ & $\begin{array}{l}\text { ATGACGTCUGATCTGACGG } \\
\text { TTCACTAAACCA }\end{array}$ \\
\hline pcDNA_backbone_fwd & $\begin{array}{l}\text { USER primer for promoter } \\
\text { donor plasmid }\end{array}$ & $\begin{array}{l}\text { ACTTGCGUACTTTTCGGGG } \\
\text { AAATGTGCG }\end{array}$ \\
\hline pcDNA_backbone_rev & $\begin{array}{l}\text { USER primer for promoter } \\
\text { donor plasmid }\end{array}$ & $\begin{array}{l}\text { ACACCGACUCAGTCGGGA } \\
\text { AACCTGTCGTG }\end{array}$ \\
\hline AAVS1_LP_fwd & $\begin{array}{l}\text { USER primer for LP donor } \\
\text { plasmid }\end{array}$ & $\begin{array}{l}\text { AAAGCCTAUATAACTTCGT } \\
\text { ATAGCATAC }\end{array}$ \\
\hline
\end{tabular}




\begin{tabular}{|c|c|c|}
\hline AAVS1_LP_rev & $\begin{array}{l}\text { USER primer for LP donor } \\
\text { plasmid }\end{array}$ & $\begin{array}{l}\text { ATGACGTCUCCATAGAGCC } \\
\text { CACCGCATC }\end{array}$ \\
\hline AAVS1_3'junction_fwd & $\begin{array}{c}\text { LP amplicon for 3' junction } \\
\text { PCR }\end{array}$ & $\begin{array}{l}\text { CCCACAACGAGGACTACA } \\
\text { CC }\end{array}$ \\
\hline PuroR_fwd & $\begin{array}{l}\text { PuroR amplicon for qRT- } \\
\text { PCR }\end{array}$ & GTCACCGAGCTGCAAGAA \\
\hline PuroR_rev & $\begin{array}{l}\text { PuroR amplicon for qRT- } \\
\text { PCR }\end{array}$ & CCGATCTCGGCGAACAC \\
\hline ETN_fwd & $\begin{array}{l}\text { USER primer for RMCE } \\
\text { donor plasmid }\end{array}$ & $\begin{array}{l}\text { AGACGTCAUATGGCGCCC } \\
\text { GTCGCCGTCT }\end{array}$ \\
\hline ETN_rev & $\begin{array}{l}\text { USER primer for RMCE } \\
\text { donor plasmid }\end{array}$ & $\begin{array}{l}\text { ACACCGACUTTATCATTTA } \\
\text { CCCGGAGACAGGG }\end{array}$ \\
\hline EPO_fwd & $\begin{array}{c}\text { USER primer for RMCE } \\
\text { donor plasmid }\end{array}$ & $\begin{array}{l}\text { AGACGTCAUCGCCACCAT } \\
\text { GGGAGTGCACG }\end{array}$ \\
\hline EPO_rev & $\begin{array}{c}\text { USER primer for RMCE } \\
\text { donor plasmid }\end{array}$ & $\begin{array}{l}\text { ACACCGACUTCATCTATCG } \\
\text { CCGGTCCGGCAAG }\end{array}$ \\
\hline loxP_lox2272_backbone_fwd & $\begin{array}{l}\text { USER primer for RMCE } \\
\text { donor plasmid }\end{array}$ & $\begin{array}{l}\text { AGTCGGTGUATAACTTCGT } \\
\text { ATAGGATAC }\end{array}$ \\
\hline loxP_lox2272_backbone_rev & $\begin{array}{l}\text { USER primer for RMCE } \\
\text { donor plasmid }\end{array}$ & $\begin{array}{l}\text { ACGCTGCTUATAACTTCGT } \\
\text { ATAATGTATGC }\end{array}$ \\
\hline TLA_CMV_fwd & $\begin{array}{l}\text { CMV specific primer for } \\
\text { TLA sequencing }\end{array}$ & $\begin{array}{l}\text { GTCAATGACGGTAAATGG } \\
\text { C }\end{array}$ \\
\hline TLA_CMV_rev & $\begin{array}{l}\text { CMV specific primer for } \\
\text { TLA sequencing }\end{array}$ & $\begin{array}{l}\text { GGGCTATGAACTAATGACC } \\
\text { C }\end{array}$ \\
\hline TLA_eGFP_fwd & $\begin{array}{l}\text { eGFP specific primer for } \\
\text { TLA sequencing }\end{array}$ & $\begin{array}{l}\text { CAACAGCCACAACGTCTAT } \\
\text { A }\end{array}$ \\
\hline TLA_eGFP_rev & $\begin{array}{l}\text { eGFP specific primer for } \\
\text { TLA sequencing }\end{array}$ & CGTCCTTGAAGAAGATGGT \\
\hline
\end{tabular}

fwd, forward primer; rev, reverse primer; TLA, targeted locus amplification 
Supporting Table S4. Promoter, GOI, and terminator sequences.

\begin{tabular}{|c|c|}
\hline Promoter & Sequence \\
\hline CMV & $\begin{array}{l}\text { CGTTACATAACTTACGGTAAATGGCCCGCCTGGCTGACCGCCCAACGACCCCCGCCCA } \\
\text { TTGACGTCAATAATGACGTATGTTCCCATAGTAACGCCAATAGGGACTTTCCATTGAC } \\
\text { GTCAATGGGTGGAGTATTTACGGTAAACTGCCCACTTGGCAGTACATCAAGTGTATCA } \\
\text { TATGCCAAGTACGCCCCCTATTGACGTCAATGACGGTAAATGGCCCGCCTGGCATTAT } \\
\text { GCCCAGTACATGACCTTATGGGACTTTCCTACTTGGCAGTACATCTACGTATTAGTCAT } \\
\text { CGCTATTACCATGGTGATGCGGTTTGGCAGTACATCAATGGGCGTGGATAGCGGTTT } \\
\text { GACTCACGGGGATTTCCAAGTCTCCACCCCATTGACGTCAATGGGAGTTTGTTTTGGC } \\
\text { ACCAAAATCAACGGGACTTTCCAAAATGTCGTAACAACTCCGCCCCATTGACGCAAAT } \\
\text { GGGCGGTAGGCGTGTACGGTGGGAGGTCTATATAAGCAGAGCT }\end{array}$ \\
\hline EF1 $\alpha$ & $\begin{array}{l}\text { GTGAGGCTCCGGTGCCCGTCAGTGGGCAGAGCGCACATCGCCCACAGTCCCCGAGAA } \\
\text { GTTGGGGGGAGGGGTCGGCAATTGAACCGGTGCCTAGAGAAGGTGGCGCGGGGTAAA } \\
\text { CTGGGAAAGTGATGTCGTGTACTGGCTCCGCCTTTTTCCCGAGGGTGGGGGAGAACCG } \\
\text { TATATAAGTGCAGTAGTCGCCGTGAACGTTCTTTTCGCAACGGGTTTGCCGCCAGAA } \\
\text { CACAGGTAAGTGCCGTGTGTGGTCCCGCGGGCCTGGCCTCTTTACGGGTTATGGCCC } \\
\text { TTGCGTGCCTTGATTACTTCCACCTGGCTCCAGTACGTGATTCTTGATCCCGAGCTGG } \\
\text { AGCCAGGGGCGGGCCTTCGCTTTAGGAGCCCTTCGCCTCGTGCTTGAGTTGAGGCC } \\
\text { TGGCCTGGGCGCTGGGGCCGCCGCGTGCGAATCTGGTGGCACCTTCGCGCCTGTCTCG } \\
\text { CTGCTTTCGATAAGTCTCTAGCCATTTAAAATTTTTGATGACCTGCTGCGACGCTTTTT } \\
\text { TTCTGGCAAGATAGTCTTGTAAATGCGGGCCAGGATCTGCACACTGGTATTTCGGTTT } \\
\text { TGGGGCCGCGGGCGGCGACGGGGCCCGTGCGTCCCAGCGCACATGTTCGGCGAGGCG } \\
\text { GGGCCTGCGAGCGCGGCCACCGAGAATCGGACGGGGGTAGTCTCAAGCTGGCCGGCC } \\
\text { TGCTCTGGTGCCTGGCCTCGCGCCGCCGTGATCGCCCCGCCTGGGCGGCAAGGCTG } \\
\text { GCCCGGTCGGCACCAGTTGCGTGAGCGGAAAGATGGCCGCTTCCCGGCCCTGCTCCAG } \\
\text { GGGGCTCAAAATGGAGGACGCGGCGCTCGGGAGAGCGGGCGGGTGAGTCACCCACAC } \\
\text { AAAGGAAAGGGGCCTTTCCGTCCTCAGCCGTCGCTTCATGTGACTCCACGGAGTACCG } \\
\text { GGCGCCGTCCAGGCACCTCGATTAGTCTGGAGCTTTTGGAGTACGTCGTCTTTAGGTT } \\
\text { GGGGGGAGGGGTTTATGCGATGGAGTTCCCACACTGAGTGGGTGGAGACTGAAG } \\
\text { TTAGGCCAGCTTGGCACTTGATGTAATTCTCCTTGGAATTGCCCTTTTGATTTGGA } \\
\text { TCTTGGTTCATTCTCAAGCCTCAGACAGTGGTTCAAAGTTTTTTCTTCCATTTCAGGT } \\
\text { GTCGTGA }\end{array}$ \\
\hline SV40 & $\begin{array}{l}\text { GTGTGTCAGTTAGGGTGTGGAAAGTCCCCAGGCTCCCCAGCAGGCAGAAGTATGCAA } \\
\text { AGCATGCATCTCAATTAGTCAGCAACCAGGTGTGGAAAGTCCCCAGGCTCCCCAGCAG } \\
\text { GCAGAAGTATGCAAAGCATGCATCTCAATTAGTCAGCAACCATAGTCCCGCCCCTAAC } \\
\text { TCCGCCCATCCCGCCCCAACTCCGCCAGTTCCGCCCATTCTCCGCCCCATGGCTGAC } \\
\text { TAATTTTTTTATTTATGCAGAGGCCGAGGCCGCCTCTGCCTCTGAGCTATTCCAGAAG } \\
\text { TAGTGAGGAGGCTTTTTGGAGGCCTAGGCTTTTGCAAAAAGCTCCCGGGAGCTTGTA } \\
\text { TATCCATTTTCGGATCTGATCAGCAC }\end{array}$ \\
\hline TK & $\begin{array}{l}\text { ATGACACAAACCCCGCCCAGCGTCTTGTCATTGGCGAATTCGAACACGCAGATGCAGT } \\
\text { CGGGGCGGCGCGGTCCCAGGTCCACTTCGCATATTAAGGTGACGCGTGTGGCCTCGAA } \\
\text { CACCGAGCGACCCTGCAGCGACCCGCTTAA }\end{array}$ \\
\hline GOI & Sequence \\
\hline PuroR & $\begin{array}{l}\text { ATGACCGAGTACAAGCCCACGGTGCGCCTCGCCACCCGCGACGACGTCCCCAGGGCC } \\
\text { GTACGCACCCTCGCCGCCGCGTTCGCCGACTACCCCGCCACGCGCCACACCGTCGATC } \\
\text { CGGACCGCCACATCGAGCGGGTCACCGAGCTGCAAGAACTCTTCCTCACGCGCGTCGG } \\
\text { GCTCGACATCGGCAAGGTGTGGGTCGCGGACGACGGCGCCGCGGTGGCGGTCTGGAC } \\
\text { CACGCCGGAGAGCGTCGAAGCGGGGGCGGTGTTCGCCGAGATCGGCCCGCGCATGGC } \\
\text { CGAGTTGAGCGGTCCCGGCTGGCCGCGCAGCAACAGATGGAAGGCTCCTGGCGCC } \\
\text { GCACCGGCCCAAGGAGCCCGCGTGGTTCCTGGCCACCGTCGGCGTCTCGCCCGCCAC } \\
\text { CAGGGCAAGGGTCTGGGCAGCGCCGTCGTGCTCCCCGGAGTGGAGGCGGCCGAGCGC } \\
\text { GCCGGGGTGCCCGCCTTCCTGGAGACCTCCGCGCCCCGCAACCTCCCCTTCTACGAGC } \\
\text { GGCTCGGCTTCACCGTCACCGCCGACGTCGAGGTGCCCGAAGGACCGCGCACCTGGTG }\end{array}$ \\
\hline
\end{tabular}




\begin{tabular}{|c|c|}
\hline & CATGACCCGCAAGCCCGGTGCCTGA \\
\hline eGFP & $\begin{array}{l}\text { ATGGTGAGCAAGGGCGAGGAGCTGTTCACCGGGGTGGTGCCCATCCTGGTCGAGCTG } \\
\text { GACGGCGACGTAAACGGCCACAAGTTCAGCGTGTCCGGCGAGGGCGAGGGCGATGCC } \\
\text { ACCTACGGCAAGCTGACCCTGAAGTTCATCTGCACCACCGGCAAGCTGCCCGTGCCCT } \\
\text { GGCCCACCCTCGTGACCACCCTGACCTACGGCGTGCAGTGCTTCAGCCGCTACCCCGA } \\
\text { CCACATGAAGCAGCACGACTTCTTCAAGTCCGCCATGCCCGAAGGCTACGTCCAGGAG } \\
\text { CGCACCATCTTCTTCAAGGACGACGGCAACTACAAGACCCGCGCCGAGGTGAAGTTC } \\
\text { GAGGGCGACACCTGGTGACCGCATCGAGCTGAAGGGCATCGACTTCAAGGAGGAC } \\
\text { GGCAACATCCTGGGGCACAAGCTGGAGTACAACTACAACAGCCACAACGTCTATATC } \\
\text { ATGGCCGACAAGCAGAAGAACGGCATCAAGGTGAACTTCAAGATCCGCCACAACATC } \\
\text { GAGGACGGCAGCGTGCAGCTCGCCGACCACTACCAGCAGAACACCCCCATCGGCGAC } \\
\text { GGCCCCGTGCTGCTGCCCGACAACCACTACCTGAGCACCCAGTCCGCCCTGAGCAAAG } \\
\text { ACCCCAACGAGAAGCGCGATCACATGGTCCTGCTGGAGTTCGTGACCGCCGCCGGGA } \\
\text { TCACTCTCGGCATGGACGAGCTGTACAAG }\end{array}$ \\
\hline mCherry & $\begin{array}{l}\text { ATGGTGAGCAAGGGCGAGGAGGATAACATGGCCATCATCAAGGAGTTCATGCGCTTC } \\
\text { AAGGTGCACATGGAGGGCTCCGTGAACGGCCACGAGTTCGAGATCGAGGGCGAGGGC } \\
\text { GAGGGCCGCCCCTACGAGGGCACCCAGACCGCCAAGCTGAAGGTGACCAAGGGTGGC } \\
\text { CCCCTGCCCTTCGCCTGGGACATCCTGTCCCCTCAGTTCATGTACGGCTCCAAGGCCTA } \\
\text { CGTGAAGCACCCCGCCGACATCCCCGACTACTTGAAGCTGTCCTTCCCCGAGGGCTTC } \\
\text { AAGTGGGAGCGCGTGATGACTTCGAGGACGGCGGCGTGGTGACCGTGACCCAGGAC } \\
\text { TCCTCCCTGCAGGACGGCGAGTTCATCTACAAGGTGAAGCTGCGCGGCACCAACTTCC } \\
\text { CCTCCGACGGCCCCGTAATGCAGAAGAAGACCATGGGCTGGGAGGCCTCCTCCGAGC } \\
\text { GGATGTACCCCGAGGACGGCGCCCTGAAGGGCGAGATCAAGCAGAGGCTGAAGCTGA } \\
\text { AGGACGGCGGCCACTACGACGCTGAGGTCAAGACCACCTACAAGGCCAAGAAGCCCG } \\
\text { TGCAGCTGCCCGGCGCCTACAACGTCAACATCAAGTTGGACATCACCTCCCACAACGA } \\
\text { GGACTACACCATCGTGGAACAGTACGAACGCGCCGAGGGCCGCCACTCCACCGGCGG } \\
\text { CATGGACGAGCTGTACAAGTAG }\end{array}$ \\
\hline ETN & 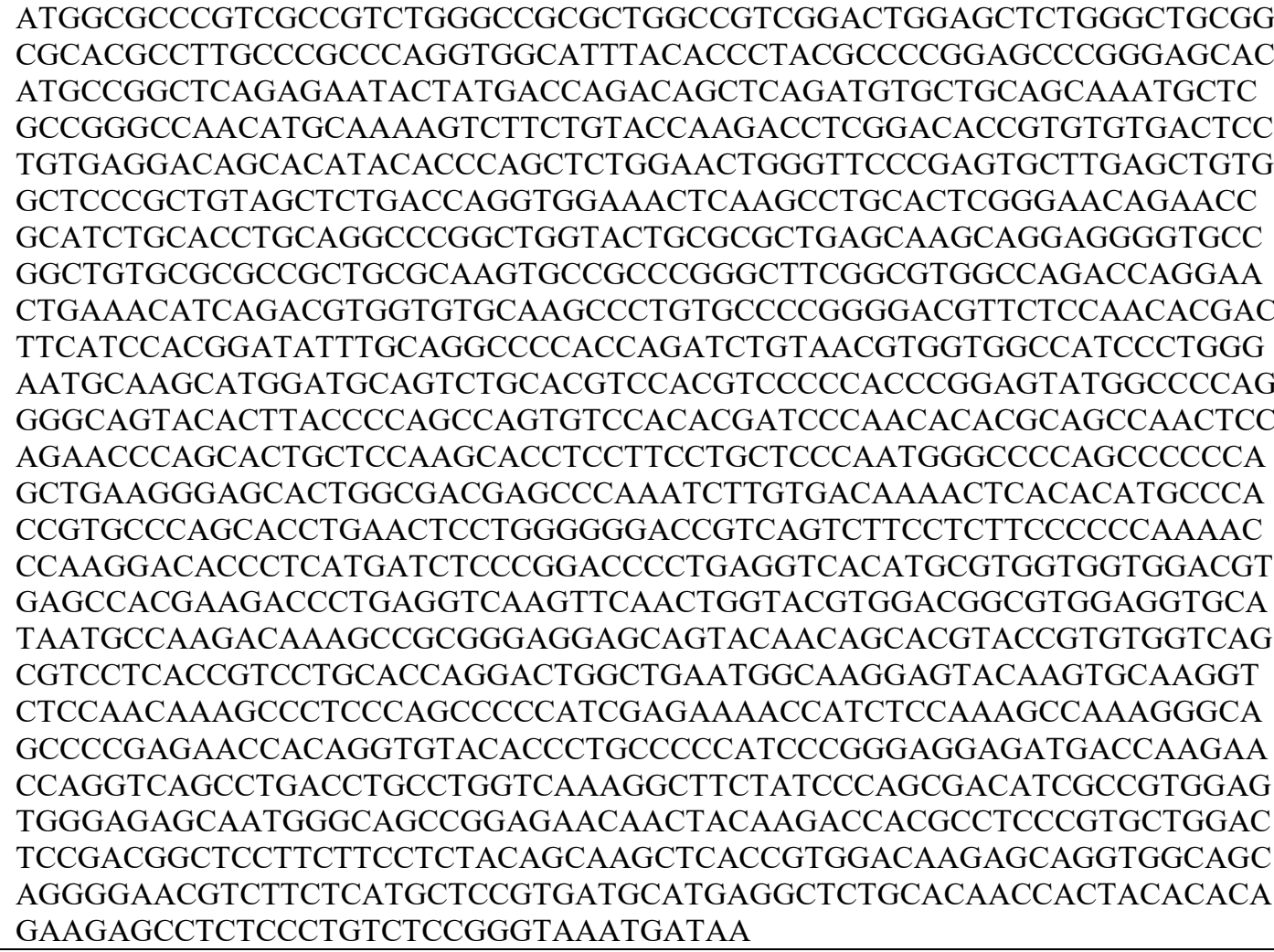 \\
\hline EPO & $\begin{array}{l}\text { ATGGGAGTGCACGAGTGTCCTGCTTGGCTGTGGCTGCTGCTGTCCCTGCTGTCTCTGCC } \\
\text { TCTGGGACTGCCTGTGCTGGGCGCTCCTCCTAGACTGATCTGCGACTCCCGGGTGCTG } \\
\text { GAAAGATACCTGCTGGAAGCCAAAGAGGCCGAGAACATCACCACCGGCTGCGCCGAG }\end{array}$ \\
\hline
\end{tabular}




\begin{tabular}{|c|c|}
\hline & $\begin{array}{l}\text { CACTGCTCCCTGAACGAGAATATCACCGTGCCCGACACCAAAGTGAACTTCTACGCCT } \\
\text { GGAAGCGGATGGAAGTGGGCCAGCAGGCTGTGGAAGTGTGGCAGGGACTGGCTCTGC } \\
\text { TGAGCGAGGCTGTGCTGAGAGGACAGGCCCTGCTCGTGAACTCCTCCCAGCCTTGGGA } \\
\text { ACCCCTGCAGCTGCACGTGGACAAGGCTGTGTCCGGCCTGAGATCCCTGACCACCCTG } \\
\text { CTGAGAGCACTGGGAGCCCAGAAAGAGGCCATCTCTCCACCTGACGCCGCCTCTGCTG } \\
\text { CTCCTCTGAGAACCATCACCGCCGACACCTTCAGAAAGCTGTTCCGGGTGTACTCCAA } \\
\text { CTTCCTGCGGGGCAAGCTGAAGCTGTACACCGGCGAGGCTTGCCGGACCGGCGATAG } \\
\text { ATGA }\end{array}$ \\
\hline SA & СTGACСТCTTCTCTTCСТCСCACAGG \\
\hline $\mathrm{T} 2 \mathrm{~A}$ & GAGGGCAGAGGAAGTCTTCTAACATGCGGTGACGTGGAGGAGAATCCCGGCCCT \\
\hline loxP & ATAACTTCGTATAGCATACATTATACGAAGTTAT \\
\hline lox2272 & ATAACTTCGTATAGGATACTTTATACGAAGTTAT \\
\hline Terminator & Sequence \\
\hline BGH poly (A) & $\begin{array}{l}\text { CTGTGCCTTCTAGTTGCCAGCCATCTGTTGTTTGCCCCTCCCCCGTGCCTTCCTTGACCC } \\
\text { TGGAAGGTGCCACTCCCACTGTCCTTTCCTAATAAAATGAGGAAATTGCATCGCATTG } \\
\text { TCTGAGTAGGTGTCATTCTATTCTGGGGGGTGGGGTGGGGCAGGACAGCAAGGGGGA } \\
\text { GGATTGGGAAGACAATAGCAGGCATGCTGGGGATGCGGTGGGCTCTATGG }\end{array}$ \\
\hline SV40 poly (A) & $\begin{array}{l}\text { AACTTGTTTATTGCAGCTTATAATGGTTACAAATAAAGCAATAGCATCACAAATTTCA } \\
\text { CAAATAAAGCATTTTTTTCACTGCATTCTAGTTGTGGTTTGTCCAAACTCATCAATGTA } \\
\text { TCTTA }\end{array}$ \\
\hline
\end{tabular}




\section{References}

(1) Lee, J. S., Kallehauge, T. B., Pedersen, L. E., and Kildegaard, H. F. (2015) Site-specific integration in CHO cells mediated by CRISPR/Cas9 and homology-directed DNA repair pathway. Sci. Rep. 5, 8572

(2) Noh, S. M., Shin, S., and Lee, G. M. (2018) Comprehensive characterization of glutamine synthetase-mediated selection for the establishment of recombinant $\mathrm{CHO}$ cells producing monoclonal antibodies. Sci. Rep. 8, 5361.

(3) Yu, Q., Katlinskaya, Y. V., Carbone. C. J., Zhao, B., Katlinski, K. V., Zheng, H., Guha, M., Li, N., Chen, Q., Yang, T., Lengner, C. J., Greenberg, R. A., Johnson, F. B., and Fuchs, S. Y. (2015) DNAdamage-induced type I interferon promotes senescence and inhibits stem cell function. Cell Rep. 11, $785-797$.

(4) Vandesompele, J., De Preter, K., Pattyn, F., Poppe, B., Van Roy, N., De Paepe, A., and Speleman, F. (2002) Accurate normalization of real-time quantitative RT-PCR data by geometric averaging of multiple internal control genes. Genome Biol. 3, research0034.

(5) Pristovšek, N., Nallapareddy, S., Grav, L. M., Hefzi, H., Lewis, N. E., Rugbjerg, P., Hansen, H. G., Lee, G. M., Andersen, M. R., and Kildegaard, H. F. (2019) Systematic evaluation of site-specific recombinant gene expression for programmable mammalian cell engineering. ACS Synth. Biol. 8, 758774.

(6) Chu, V. T., Weber, T., Wefers, B., Wurst, W., Sander, S., Rajewsky, K., and Kühn, R. (2015) Increasing the efficiency of homology-directed repair for CRISPR-Cas9-induced precise gene editing in mammalian cells. Nat. Biotechnol. 33, 543-548.

(7) Chen, X., Janssen, J. M., Liu, J., Maggio, I., 't Jong, A. E. J., Mikkers, H. M. M., and Gonçalves, M. A. F. V. (2017) In trans paired nicking triggers seamless genome editing without double-stranded DNA cutting. Nat. Commun. 8, 657. 
(8) Bertero, A., Pawlowski, M., Ortmann, D., Snijders, K., Yiangou, L., Cardoso de Brito, M., Brown, S., Bernard, W. G., Cooper, J. D., Giacomelli, E., Gambardella, L., Hannan, N. R., Iyer, D., Sampaziotis, F., Serrano, F., Zonneveld, M. C., Sinha, S., Kotter, M., and Vallier, L. (2016) Optimized inducible shRNA and CRISPR/Cas9 platforms for in vitro studies of human development using hPSCs. Development 143, 4405-4418. 